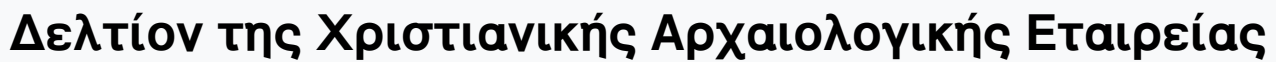

Tó 20 (1999)

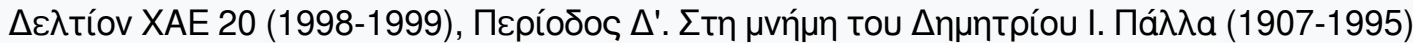

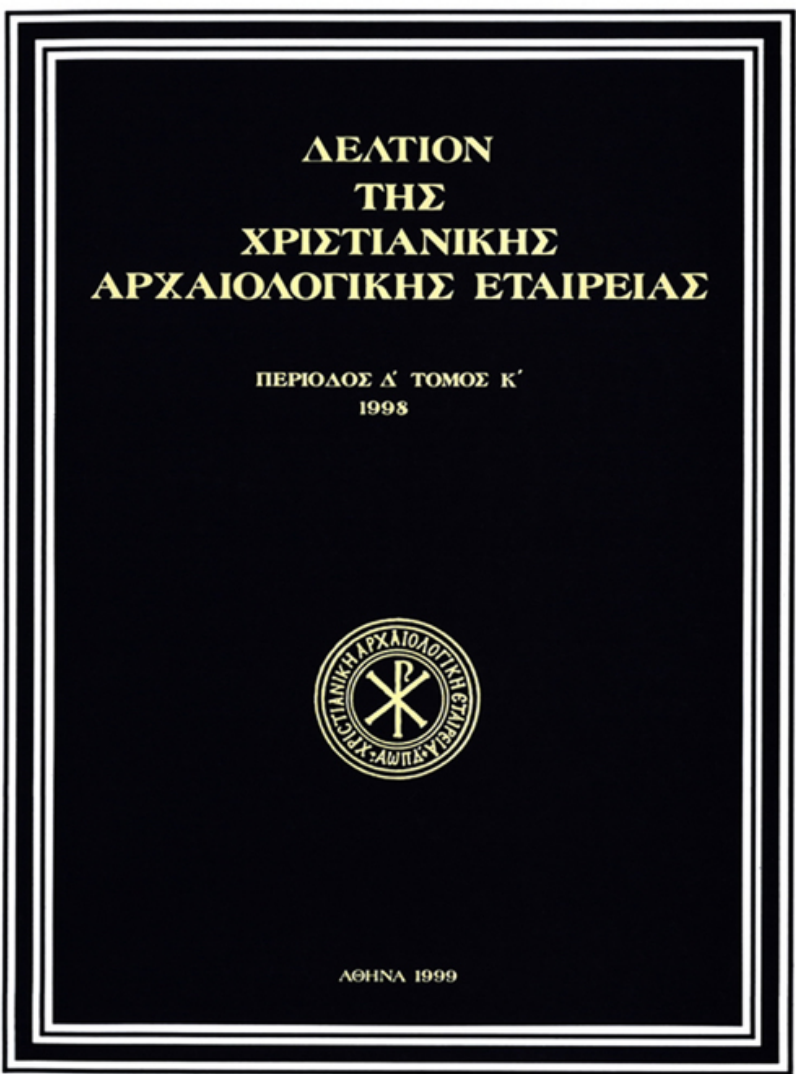

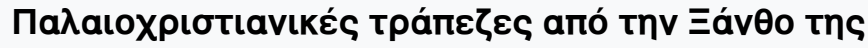
Nukías

Jean-Pierre SODINI

doi: $\underline{10.12681 / \text { dchae. } 1190}$

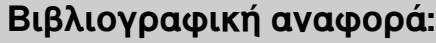

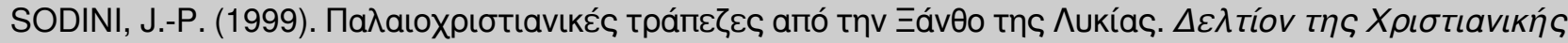
Архаıолоүıки́s Eтаıрві́as, 20, 41-46. https://doi.org/10.12681/dchae.1190 


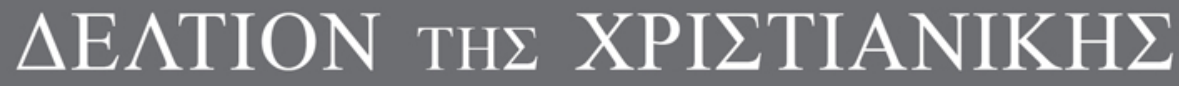 APXAIO $\Lambda$ OГIKH $\Sigma$ ETAIPEIA $\Sigma$}

Tables de Xanthos

Jean-Pierre SODINI

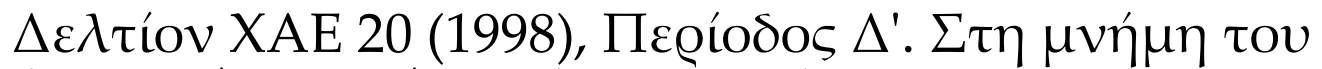

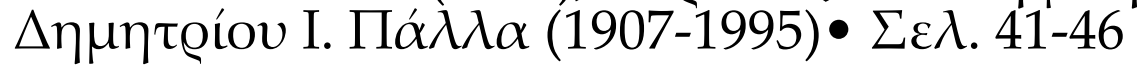

A@HNA 1999

EONIKO KENTPO 


\section{Jean-Pierre Sodini}

\section{TABLES DE XANTHOS}

L a fouille de la basilique orientale de Xanthos, commencée au début des années 70 dans la cadre de la mission française de Xanthos-Letôon ${ }^{1}$, a livré un nombre important de pièces sculptées. Sans parler des remplois, les unes sont en marbre, importées de Proconnèse ou d'autres carrières (chapiteaux corinthiens ${ }^{2}$, colonnes, bases, placages et dalles de pavement, quelques plaques et piliers de chancel), les autres en calcaire local, notamment un calcaire tendre travaillé dans un style régional très particulier (la majorité des plaques et piliers de chancel et des parapets d'escalier caractéristiques des ambons, de très nombreuses corniches ou éléments apparentés) $)^{3}$. La Lycie, contrairement aux rives du bassin égéen, fait partie des régions où le « tout marbre " dans la sculpture architecturale se fait rare en raison de la distance, sinon des carrières, du moins des ateliers produisant la sculpture utilisée dans les églises. On compte parmi elles la Pamphylie, la Cilicie, la Crète, Chypre, le littoral syro-palestinien. L'importance du marbre recule encore dès que l'on quitte les zones côtières ou dès que l'on aborde des rives plus éloignées de la Méditerranée. Toutefois, même là, arrivent d'importantes cargaisons destinées à souligner le prestige des édifices (Ravenne) ou à accompagner la propagande impériale (Cyrénaïque, Tripolitaine et Afrique Proconsulaire).

1. Je remercie les directeurs successifs de la mission, H. Metzger, C. Le Roy, J. des Courtils de leur confiance et de leur aide, le Ministère des Affaires Etrangères qui subventionne la fouille ainsi que les autorités archéologiques turques. Les dessins ont été faits par Marie Pat Raynaud qui m'a beaucoup aidé dans l'étude des fragments et leurs collages.

2. J.-P. Sodini, Une iconostase byzantine à Xanthos, Actes $d u$ Colloque sur la Lycie antique, Paris 1980, p. 119-148, particulièrement p. 28-29, pl. XXXVe-f.

3. J.-P. Sodini, Sculptures lyciennes d'époque paléochrétienne à représentations humaines et animalières, $R A$ 1976, p. 337-348 ; R.M. Harrison, Churches and Chapels of Central Lycia, AnatSt 13 (1963), p. 117-151; id., A Note on Architectural Sculpture in Central Lycia, AnatSt 22 (1972), p. 187-197 ; id., Nouvelles découvertes romaines
Dans presque toutes les églises utilisant un matériau «mixte » en sculpture, les tables d'autel ou liturgiques sont en marbre, qui est la pierre la plus noble et qui constitue un « Ersatz » de l'argent, ainsi qu'un texte bien connu de la Vie de Théodore de Sykeôn ${ }^{4}$ le laisse entendre et comme on peut le comprendre facilement d'après l'imitation flagrante de l'argenterie dans certains décors de table. L'importance des travaux de D.I. Pallas sur les rapports entre liturgie et architecture, ses recherches sur l'autel ( « thalassa $»^{5}$ et tables ${ }^{6}$ ), m'amènent tout naturellement à lui faire hommage de certaines tables trouvées dans les fouilles effectuées dans l'église Est de Xanthos.

\section{A. Tables rectangulaires}

1. Plateau à tranche moulurée : boudin médian encadré de deux listels

Marbre blanc de grain moyen à fin. Deux fragments non jointifs ont dû appartenir à la même table d'une épaisseur au rebord de $9,5 \mathrm{~cm}$ et au centre de $5 \mathrm{~cm}$. Le revers est parfaitement lisse.

a) C1 (Fig. 1) : $69 \times 40,5 \mathrm{~cm}$. Sur le rebord, fragment de croix ; sur le plateau, restes d'enroulements avec feuilles

tardives et paléobyzantines, CRAI 1979, p. 222-239 ; J. Borchhardt, Myra, Berlin 1975, p. 359-428 (O. Feld, essentiellement Saint-Nicolas de Demre). Depuis 1989, Y. Ötuken a entrepris des fouilles dans l'église Saint-Nicolas, au cours desquelles un grand nombre de sculptures ont été découvertes qui sont en cours de publication: cf. en dernier lieu, Recherches en Lycie centrale et fouilles dans l'église Saint-Nicolas (en turc), Adalya 1 (1996), p. 73-85.

4. A.-J. Festugière, Vie de Théodore de Sykeôn, SubHGr 48, Bruxelles 1970, par. 42, I, p. 36, II, p. 39.

5. D.I. Pallas, La «thalassa» dans l'église chrétienne. Contribution à l'histoire de l'autel chrétien à la morphologie de la liturgie (en grec), Athènes 1952.

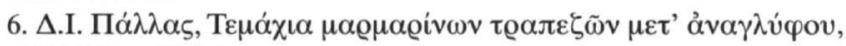
$A E$ 1930, p. 90-96. 


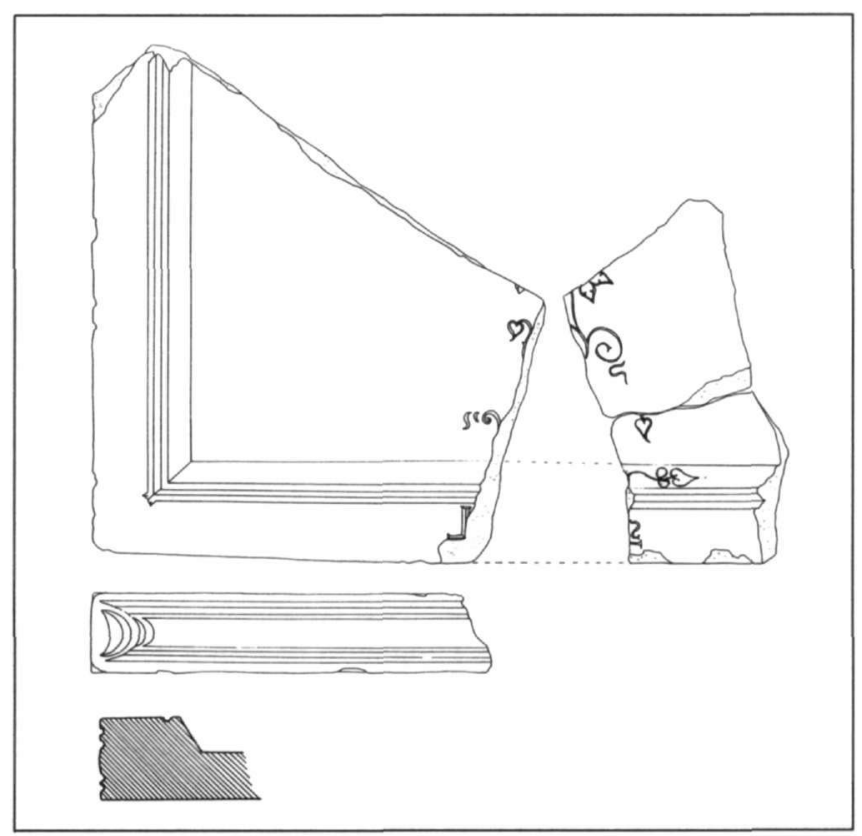

Fig. 1. Fragments de table C1, C3 + C10 (M.-P. R.).

cordiformes et vrille. Sur la tranche, l'angle est marqué par la lunule si souvent observée ${ }^{7}$.

b) $\mathrm{C} 3+\mathrm{C} 10$ (Fig. 1 et 2) : $46 \times 21,5 \mathrm{~cm}$. Même décor que sur le fragment précédent : sur le rebord, croix avec rho à l'extrémité supérieure ; sur le plateau, enroulements, vrilles et feuilles dont une au moins s'apparente davantage à une feuille de vigne qu'à une feuille cordiforme (lierre?). La place relative des deux fragments n'est pas calculable : la présence des mêmes motifs peut sans doute indiquer que le fragment $b$ se trouvait dans le prolongement du bord décoré du fragment a.

A ces deux fragments, peut sans doute être joint un troisième, $\mathrm{C} 2$ (Fig. 3) : $68 \times 41,5 \mathrm{~cm}$; même marbre, même épaisseur. Le tore médian est un peu plus étroit. Peut-être est-ce une partie d'un troisième côté de la même table ?

La combinaison d'un boudin ${ }^{8}$ et de deux listels sur la

7. Donnons quelques exemples récents: R.M. Bonacasa Carra, Il complesso paleocristiano a nord del teatro di Sabratha, Quad. Arch. Libya 14 (1991), p. 198, B et C, fig. 95c et 96e, et ead., Marmi dall'arredo liturgico delle chiese di Sabratha, Quad. Arch. Libya 15 (1992), p. 312, fig. 9 et p. 314, fig. 12-13 (Sabratha, bas. 3) ; N. Duval, C. Metzger, D. Feissel, Tables et reliquaires du Louvre, Recueil du Musée National de Belgrade 16-1 (1996), p. 311-314, où l'on trouvera la bibliographie principale.

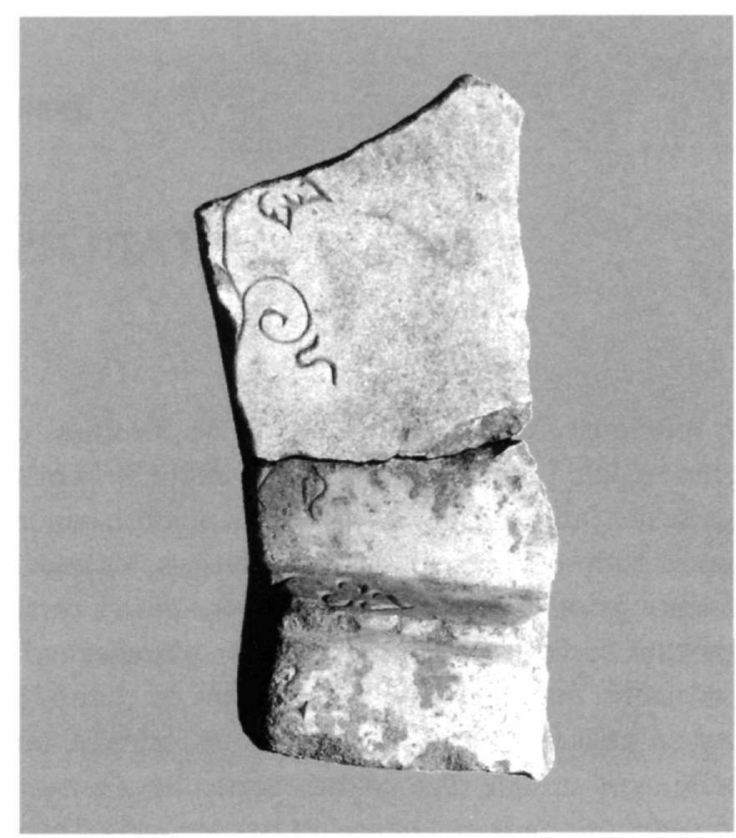

Fig. 2. Fragment $C 3+C 10$.

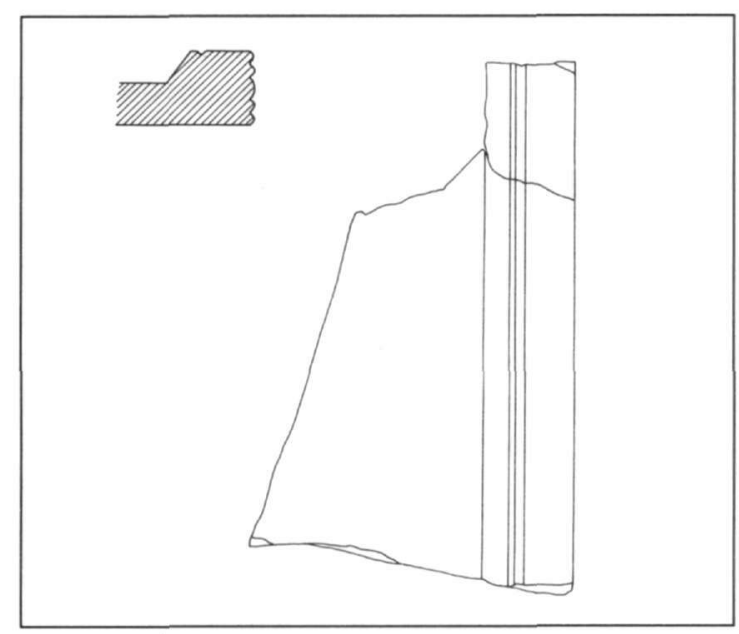

Fig. 3. Fragment C2. (M.-P. R.).

8. Boudin simple à Saint-Isidore de Chio : 'A.K. 'O@ $\lambda \alpha \dot{\alpha} v \delta o \zeta, ~ ' H \xi v \lambda$ ó$^{-}$

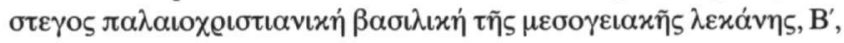
Athènes 1954 (cité ensuite: Orlandos, Basilique), p. 450, fig. 409.3; on peut y adjoindre Salone (N. Duval, E. Marin, C. Metzger, Salona I, Catalogue de la sculpture architecturale paléochrétienne de Salone, Rome-Split 1994 (cité ensuite : Salona I), pl. L, VIg 1) et Saint-Jean l'Evangéliste à Ravenne, bien que l'on n'ait pas de relevé détaillé de cette moulure : Orlandos, Basilique, fig. 409.1. 


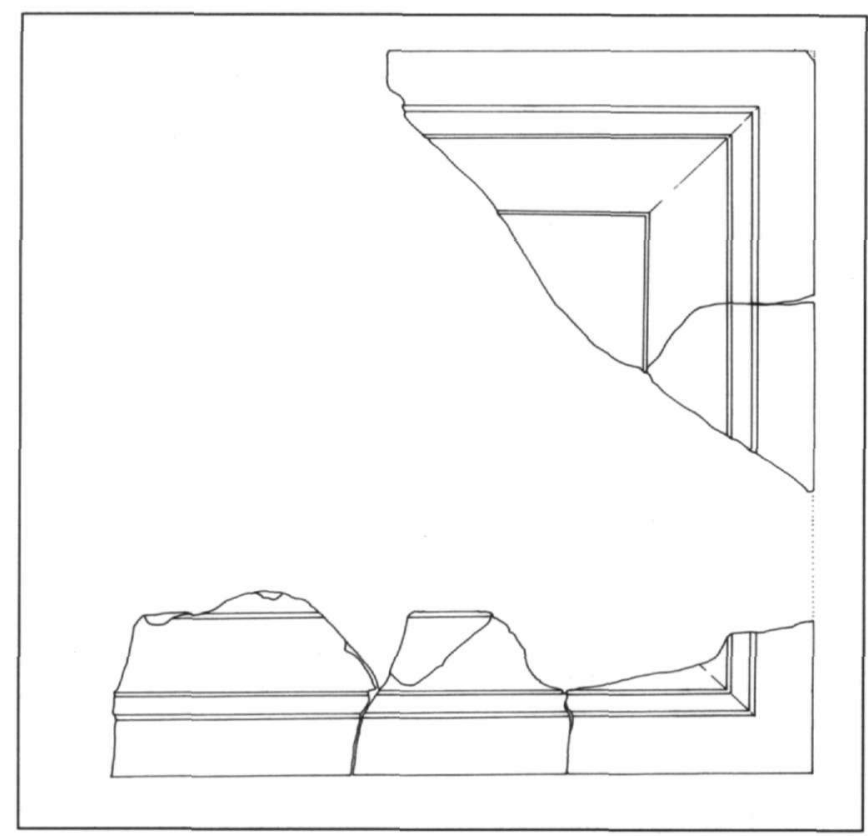

Fig. 4. Fragments C4 et C5 (M.-P. R.).

tranche est complexe et ne correspond pas au type courant, de même que le chanfrein de raccord entre rebord et plateau au lieu de la courbe concave attendue. Le décor incisé d'enroulements végétaux est rare sur les tables rectangulaires. On en trouve, beaucoup plus élaboré, traité en champlevé, avec vigne habitée sortant d'un canthare axial, sur une table en sigma de Nea Anchialos ${ }^{9}$. La longueur et la largeur de la table ne sont pas déterminables. Il s'agit d'une grande table d'autel, lourde et probablement de taille importante, sans doute portée par plus de quatre pieds ${ }^{10}$. Il ne subsiste dans le sanctuaire de l'église aucun emplacement des pieds.

\section{Plateau à tranche biaise non moulurée}

Marbre jaune clair, translucide. Deux fragments non jointifs présentent une épaisseur au rebord de $6,5 \mathrm{~cm}$ et au plateau de $3 \mathrm{~cm}$. Le bord mouluré présente de l'extérieur vers l'intérieur un large bandeau plat, un onglet, un listel qui se

9. E. Chalkia, Le mense paleocristiane, Vatican 1991, p. 77, fig. 64, type I : Gr. 1

10. Orlandos, Basilique, p. 447, fig. 405.

11. Duval, Marin, Metzger, Salona I, pl. XXVII (VIIa 8), pl. LV (VIIg 26 et 27) et pl. LVII (VIIg 19), ces trois derniers exemples avec tranche biaise.

12. Chalkia, op.cit., p. 54-55, type F : Gr. 1.

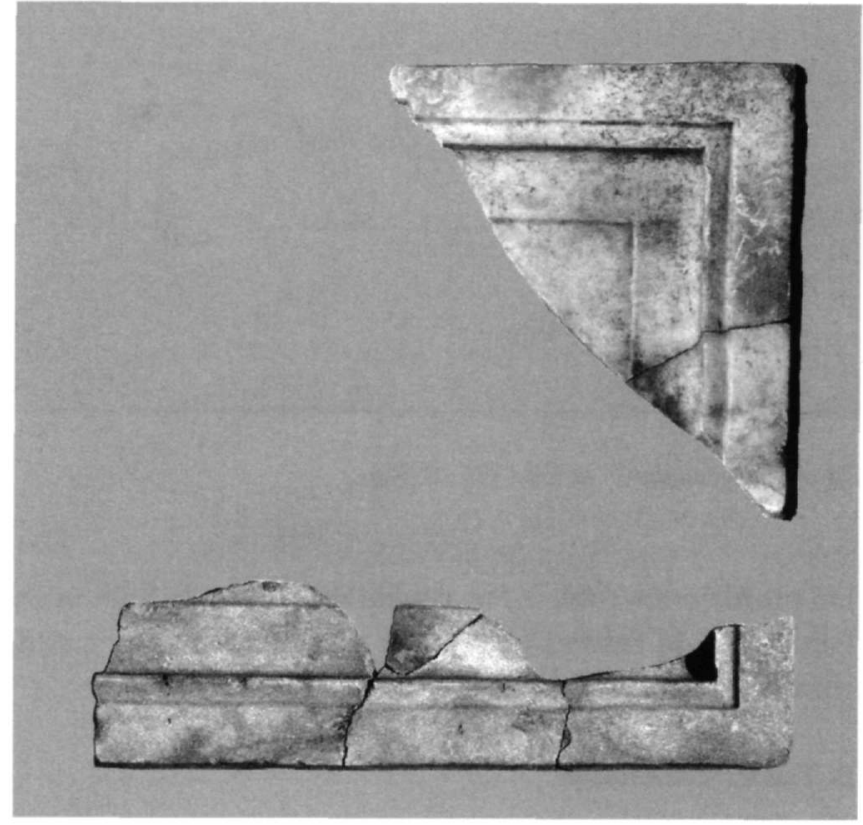

Fig. 5. Fragments C4 et C5.

raccorde au plateau par un congé abrupt. Un bandeau en légère surépaisseur encadre la partie centrale du plateau. La tranche n'est pas tout à fait droite mais biaise. Le revers est poli.

a) $\mathrm{C} 4$ (Fig. 4 et 5): $76 \times 21 \mathrm{~cm}$.

b) C5 (Fig. 4 et 5) : $50 \times 46,5 \mathrm{~cm}$.

Ce profil est plus fréquent que le précédent. Il est attesté à Salone $^{11}$, à Philippes ${ }^{12}$. Les dimensions ne sont pas restituables mais devaient être inférieures à celles de la première.

3. Plateau à tranche incurvée non moulurée (Fig. 6, 7 et 8) Marbre gris de grain moyen à gros. Deux fragments de petite taille (C7 : angle, $17 \times 12,5 \mathrm{~cm} ; \mathrm{C} 11: 17 \times 13 \mathrm{~cm})$ non jointifs. Epaisseur du rebord, $7 \mathrm{~cm}$, du plateau, $4,5 \mathrm{~cm}$. Le bord offre un méplat périphérique très étroit $(2,5 \mathrm{~cm})$, un onglet, un listel. Un court biseau racorde le rebord au plateau, suivi d'un bandeau en surépaisseur de $5 \mathrm{~cm}$. Le revers présente des traces caractéristiques de gradine ${ }^{13}$.

13. J.-Cl. Bessac, L'outillage traditionnel du tailleur de pierre, Paris 1986 , fig. 32 et p. 139-143 ; cf. aussi id., Traces d'outils sur les pierres : quelques repères chronologiques, Actes $d u$ Xe Colloque International de Glyptographie du Mont-Sainte-Odile, Braine-le-Château 1997, p. 732 (voir notamment les photos des traces d'outils obtenues par des répliques modernes, p. 28, fig. 7). 


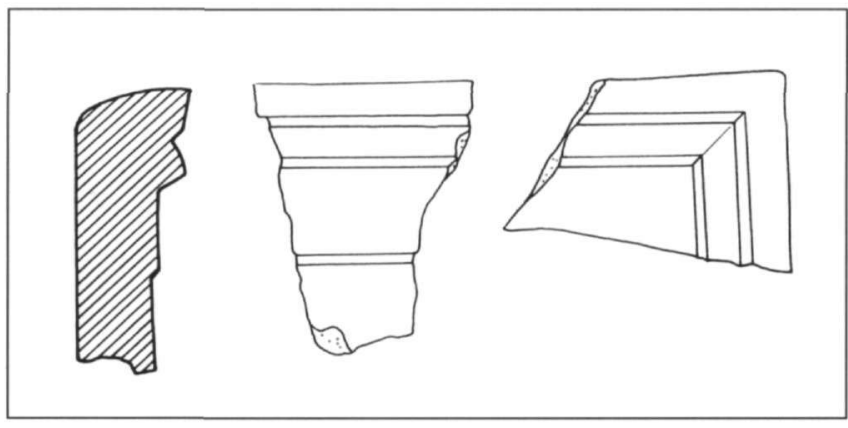

Fig. 6. Fragments C7 et C11 (M.-P. R.).

Un profil comparable a été signalé au Musée de Split mais l'épaisseur du rebord $(11 \mathrm{~cm})$ et du fond $(7,5 \mathrm{~cm})$ paraît considérable et a fait douter de son identification ${ }^{14}$.

\section{B. Tables circulaires}

1. Tables " en bec de corbin"

a) C8 (Fig. 9 et 10). Marbre jaunâtre, très fin, très translucide. Le diamètre de la table est difficile à déterminer, $c a$ $1 \mathrm{~m}$. Dimensions conservées : $28 \times 8 \mathrm{~cm}$; la hauteur est de 7 $\mathrm{cm}$; épaisseur du plateau, $2 \mathrm{~cm}$. Revers un peu rugueux.

b) C9 (Fig. 11). Marbre identique. Dimensions conservées : $15 \times 9 \mathrm{~cm}$; hauteur conservée, $8 \mathrm{~cm}$; épaisseur du plateau, $1,5 \mathrm{~cm}$. Une croix a été incisée sur le rebord. Juste à gauche de celle-ci, une cavité d'évacuation peut avoir été creusée après coup, peut-être lors de la mise en oeuvre de la table (Fig. 12) ${ }^{15}$. Le revers présente des restes d'empreintes de ciseau denté en partie ravalées, mais aussi une curieuse ligne difficile à interpréter (Fig. 13). Elle semble marquer la limite d'un surcreusement et, si elle n'est pas accidentelle, pourrait correspondre aux supports de la table. La petite taille du fragment et l'absence de parallèles ne permettent guère d'aller au-delà.

Les tables en bec de corbin sont l'une des catégories les mieux attestées parmi les tables circulaires ${ }^{16}$. Le profil et la qualité exceptionnelle du marbre indiquent vraisemblablement pour la plupart des tables de ce type la production

14. Duval, Marin, Metzger, Salona I, pl. LV (VIIg 24).

15. Le seul parallèle que je connaisse se trouve dans une table rectangulaire de Salone: Duval, Marin, Metzger, Salona I, p. 124-125, pl. XXV (VIIa 2).

16. Chalkia, op.cit. (n. 9), p. 53 et catalogue p. 195-215 (ensemble des tables circulaires).

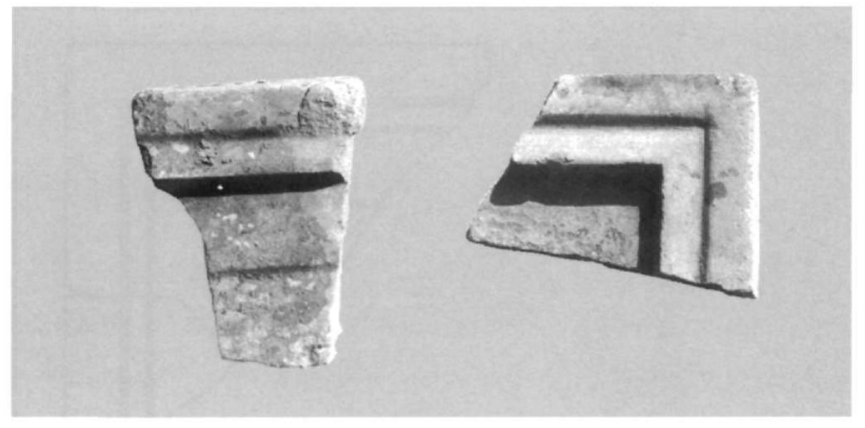

Fig. 7. Fragments $C 7$ et $C 11$, face.

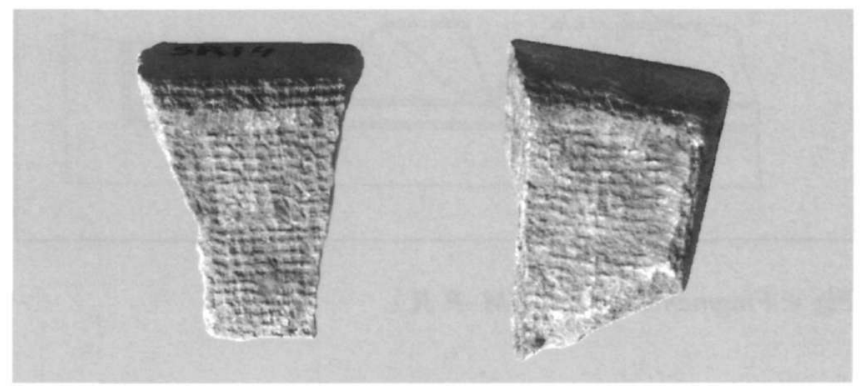

Fig. 8. Mêmes fragments, revers.

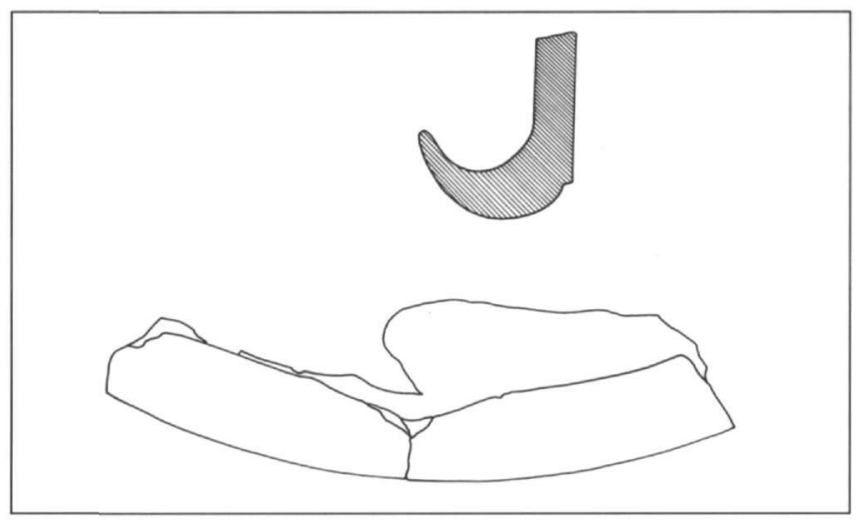

Fig. 9. Fragment C8 (M.-P. R.).

Fig. 10. Fragment C8.

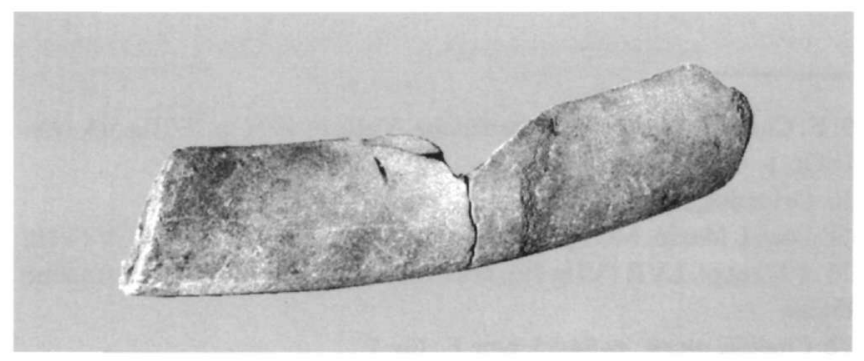




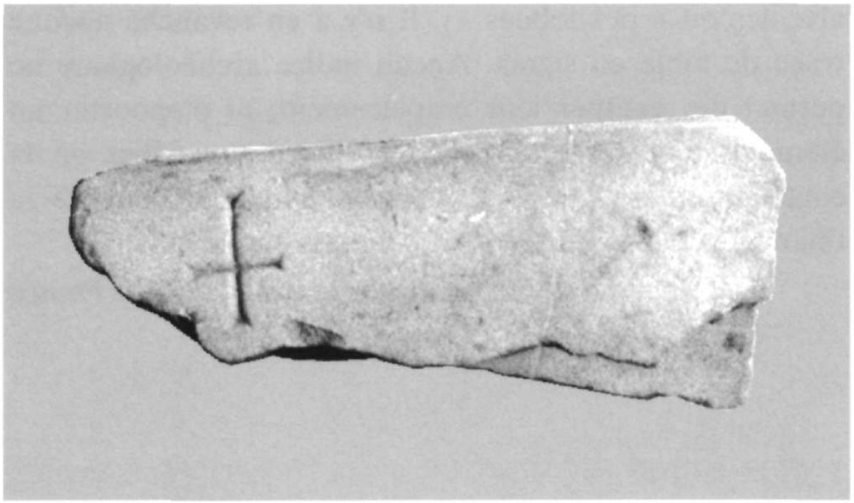

Fig. 11. Fragment C9, vue d'en haut.

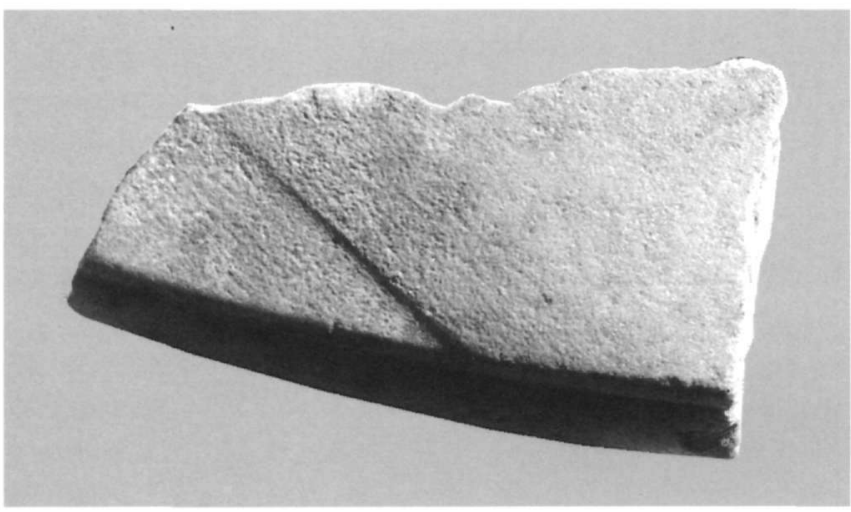

Fig. 13. Même fragment, revers.

spécifique d'un atelier, sans doute reprise et imitée pour quelques autres exemplaires dans un autre matériau et avec une exécution plus médiocre.

\section{Fragment de plateau à support annulaire (Fig. 14 et 15)}

C85. Marbre blanc à grains moyens. Le diamètre de l'anneau devait être compris entre 60 et $80 \mathrm{~cm}$. Le fragment conservé a comme dimension maximale $15,2 \mathrm{~cm}$ et comme épaisseur $1,8 \mathrm{~cm}$. La forme du rebord est inconnue. La caractéristique du fragment est la présence des stries rayonnantes qui semblent indiquer un travail à la pointe, qui sont arrêtées à $2,5 \mathrm{~cm}$ de l'anneau par des stries concentriques à ce dernier. A l'extérieur de l'anneau les stries rayonnantes semblent adoucies par un travail de polissage.

On pourrait penser que ces stries correspondent à une partie du fond qui était destiné à être caché par le support, l'anneau servant à emboiter en quelque sorte le plateau à

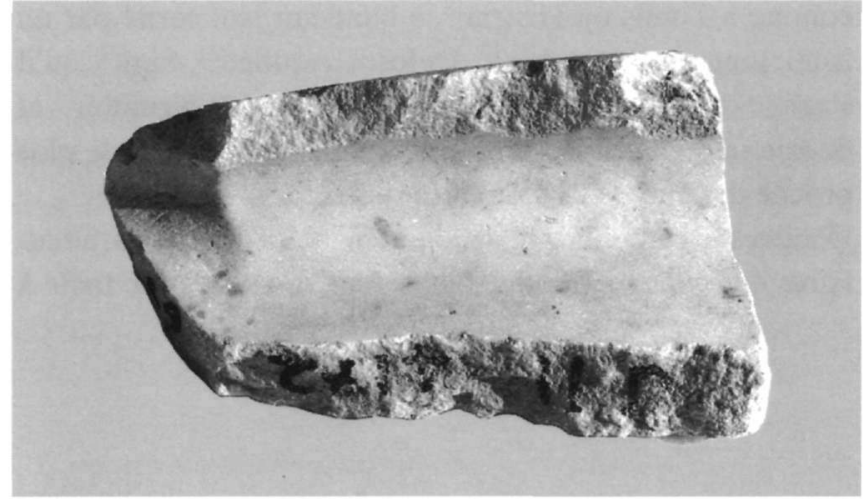

Fig. 12. Même fragment, vue frontale: à g., cavité en entonnoir.

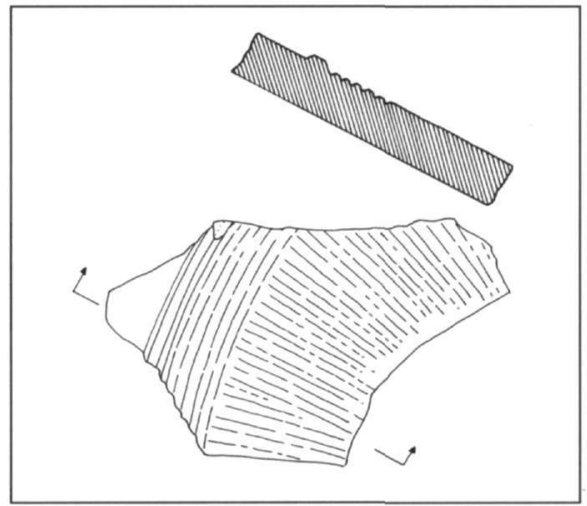

Fig. 14. Fragment C85 (M.-P. R.).

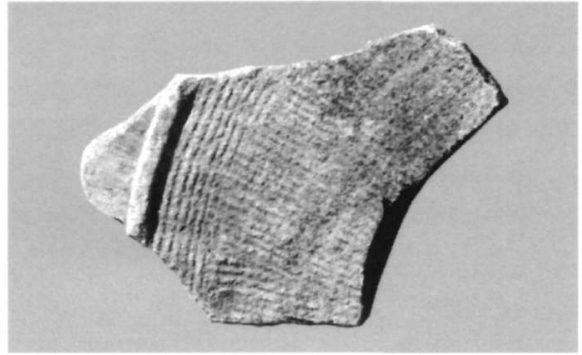

Fig. 15. Même fragment, revers.

son support ${ }^{17}$. Ce travail est caractéristique d'un certain nombre de tables circulaires, qu'elles soient à bec de corbin,

17. J. Marcadé et G. Roux, Tables et plateaux chrétiens en marbre découverts à Delphes, Etudes Delphiques (BCH Suppl. IV), Paris 1977, p. $453-465$. 
comme à Tomis ou Histria ${ }^{18}$, à bandeau plat cerné par un listel, comme à Delphes ${ }^{19}$, Délos $^{20}$, Aquilée $^{21}, \mathrm{Sinj}^{22}$, qu'il s'agisse des mensae funéraires ou piscinae de Sirmium ${ }^{23}$ et de Salone ${ }^{24}$. Le traitement observé à Tomis semble le plus proche de celui de l'exemplaire xanthien.

D'autres fragments appartenant à d'autres tables circulaires ont également été trouvés (toutefois, aucune table à alvéoles ou « polylobées »). Il n'y a en revanche aucune trace de table en sigma. Aucun indice archéologique ne permet de restituer leur emplacement, ni d'apporter un élément neuf sur leur fonction liturgique, débat où la contribution de D.I. Pallas, excellent connaisseur de la théologie et de la liturgie, avait été très importante.

Université de Paris-I, Institut Universitaire de France
18. N. Duval, L'archéologie chrétienne en Roumanie. A propos de deux livres récents d'I. Barnea, $R A$ 1980, p. 338, fig. 14b et d.

19. Marcadé et Roux, op.cit. (n. 17), fig. 11 et p. 459.

20. G. Roux, Problèmes déliens, BCH 105 (1981), p. 77-78, fig. 24.

21. N. Duval, Piscinae et mensae funéraires de Salone à Aquilée, Ant. Altoadriatiche 26 (1983), p. 443 et fig. 7 (Sirmium plutôt qu'Aquilée?).

22. Dernière publication : Duval, Marin, Metzger, Salona I, pl. LXI
(VIIh 8).

23. N. Duval, Mensae funéraires de Sirmium et de Salone, Bull. Arch. Hist. Dalmate 77 (1984), p. 192, fig. 6, pl. XXVII.1 et 2, auxquels il faut ajouter des fragments de Zagreb (ibid., p. 195-196, pl. XXVII.4 et 6). 24. Duval, Marin, Metzger, Salona I, pl. LX (VIIh 12 et 14), pl. XXXIII (VIIc 1 et 2 + A 38 et A 3948), pl. XXXIV (VIIc 3). 